\title{
Electronic Energy Loss of the Partially Stripped Boron-Like and Carbon-Like Fast Ions
}

\author{
H. GüMüss', C. ÖZALP ANd A. KÖROĞLU \\ Department of Physics, Faculty of Science and Arts \\ Ondokuz Mayıs University, Samsun, Turkey
}

(Received May 10, 2002; revised version October 1, 2002)

\begin{abstract}
An analytical formula of the electronic stopping power expression in this study was derived for swift boron-like and carbon-like ions by using first-order perturbation theory and frozen-charge-state model. The HartreeFock-Slater determinant was used for the description of the bound electrons attached to ions in the ground state and orbital-screening parameter was determined by variational method. The calculated ground state energies in this study were compared with the results of Clementi-Roetti and they are in good agreement with $5 \%$. It has been observed that the difference of energy loss for boron-like and carbon-like projectiles in a frozen-charge state increases as an atomic number increases. Furthermore, the analytical expression of the effective charge of boron-like and carbon-like projectiles was derived.
\end{abstract}

PACS numbers: $34.50 . \mathrm{Bw}, 61.85 .+\mathrm{p}$

\section{Introduction}

In the interaction of swift charged particles passing through matter, the quantity of stopping power or energy loss plays the main role in many fields such as impurity atom implanting to semiconductor device, structural analysis of solid target by Rutherford backscattering spectroscopy (RBS), and plasma-first wall interactions in nuclear-fusion reactor.

* corresponding author; e-mail: hgumus@omu.edu.tr 
The electronic stopping power of materials has been extensively investigated for fast-ionised projectiles, based on the first Born approximation. In general, stopping power data for gases and solid media were provided from the experimental view point $[1,2]$. On the other hand, theoretical studies have been based on the atomic model [3, 4], the free-electron gas model [5-7], kinetic model [8], a nonlinear calculation [9], the local density model [10,11], and the wave-packet model [12].

As a quantum-mechanical, Bethe and Bloch first theoretically studied the quantity of stopping power considering the point charge state [3, 4]. For a swift point charge $Z_{1} e$ moving at a velocity $v$ in a target matter with an atomic number $Z_{2}$, the energy loss per unit path length is formulated [3] by

$$
S=\left(\frac{4 \pi e^{4}}{m v^{2}}\right) N Z_{2} Z_{1}^{2} \ln \left(\frac{2 m v^{2}}{I}\right),
$$

where $m$ and $e$ are the electron rest mass and the elementary charge and $N$ and $I$ - the number density and the mean excitation energy of the target atoms, respectively. Later, the higher-order terms, the Barkas term (or $Z_{1}^{3}$ term) [13] and the Bloch term (or $Z_{1}^{4}$ term) [4], together with shell corrections [14] were added to the Bethe expression to obtain the standard formula for energy loss of fully stripped swift light ions.

The energy loss of partially stripped ions for a slow $\mathrm{He}^{+}$ion passing through degenerated electron gas was firstly investigated by Ferrell and Ritchie [15]. If the number of bound electrons on projectile is large enough, the partially stripped ions may be just explained by a Thomas-Fermi atom model and then its energy loss in the electron gas can be similarly formulated $[16,17]$ as the treatment of Ferrell and Ritchie. Furthermore, the concept of effective charge $Z_{\text {eff }}$ is necessary to define as the square root of the stopping power for projectile relative to that for proton moving in the same matter at the same velocity. Two quantities as the spatial size and average number of bound electron generally contribute to the effective charge.

For the partially stripped fast hydrogen-like and helium-like ions, an analytical formula for electronic stopping power was derived by Kaneko [18]. Later on, he extended this analytical formula to $\xi \leq 4$ bound electron [19]

$$
S=\left(\frac{4 \pi e^{4}}{m v^{2}}\right) N Z_{2} L\left(Z_{1}, Z_{2}, v\right)
$$

with

$$
\begin{aligned}
& L\left(Z_{1}, Z_{2}, v\right)=\left(Z_{1}-N_{2 s}-2\right)^{2} \ln \left(\frac{2 m v^{2}}{I}\right) \\
& +\left(2 Z_{1} N_{2 s}+\frac{512}{81} N_{2 s}-N_{2 s}^{2}\right) \ln \left(\frac{2 v}{Z_{e} v_{0}}\right)+4\left(Z_{1}-1-\frac{209}{81} N_{2 s}\right) \ln \left(\frac{v}{Z_{e} v_{0}}\right) \\
& +2 Z_{1}+52 Z_{1} N_{2 s}-\frac{334}{27} N_{2 s}-113-\frac{271}{168} N_{2 s}^{2} .
\end{aligned}
$$


Here the Barkas and Bloch terms and the shell correction are neglected. In Eq. (2b) $N_{2 s}$ denotes the number of $2 s$ electron. $Z_{e}=Z_{\mathrm{Li}}$ and $N_{2 s}=1$ for lithium-like projectiles, $Z_{e}=Z_{\mathrm{Be}}$ and $N_{2 s}=2$ for beryllium-like ones. The quantity $Z_{e}$ is the orbital screening parameter, $v_{0}$ is the Bohr velocity $\left(2.188 \times 10^{8} \mathrm{~cm} / \mathrm{s}\right)$.

In this study, analytical formulae for the electronic energy loss of swift boron-like and carbon-like ions are presented within the framework of the first Born approximation. Firstly, total energy is minimised using a variational method in order to determine the spatial distribution of the bound electrons on projectile. Secondly, analytical formula for the stopping power is derived for swift boron-like and carbon-like ions and a degree of screening per bound electron is discussed.

\section{Theory}

In the present work, we consider the case where the projectile velocities are higher than both the statistical average velocity $Z_{2}^{2 / 3} v_{0}\left(v_{0}=2.188 \times 10^{8} \mathrm{~cm} / \mathrm{s}\right)$ of the target electron and the velocity $Z_{e} v_{0}$ of bound electrons on the projectile. Here, our procedure is based on the first-order perturbation treatment, so that the formulae derived later for swift partially stripped ions correspond to the Bethe expression (1) in the sense of having the same theoretical base [18, 19]. The spatial distribution of the bound electrons on a projectile is determined by minimising total energy, using a variational method.

Here we consider the electron distribution $\rho(r)$ in the ground state projectile under the frozen charge state. We consider a boron-like projectile having five electrons in the $1 s^{2} 2 s^{2} 2 p$ singlet configuration and a carbon-like projectile having six electrons in the $1 s^{2} 2 s^{2} 2 p^{2}$ configuration, respectively. In order to calculate the total energy of system in a quantum mechanical manner, the Hamiltonian of our system is given as

$$
H=\sum_{i}\left(-\frac{\hbar^{2}}{2 m} \nabla_{i}^{2}-\frac{Z_{1} e^{2}}{r_{i}}\right)+\frac{1}{2} \sum_{i(i \neq j)} V_{i j},
$$

where $V_{i j}$ is the interaction potential energy of two electrons in $i$ and $j$ positions and written as

$$
V_{i j}=\frac{e^{2}}{\left|r_{i}-r_{j}\right|}
$$

The wave function $\Psi$ of carbon-like particles are described by Hartree-Fock-Slater determinant:

$$
\Psi=\frac{1}{\sqrt{6 !}}\left|\begin{array}{llllll}
\psi_{1 s}\left(\boldsymbol{r}_{1}\right) \alpha & \psi_{1 s}\left(\boldsymbol{r}_{1}\right) \beta & \psi_{2 s}\left(\boldsymbol{r}_{1}\right) \alpha & \psi_{2 s}\left(\boldsymbol{r}_{1}\right) \beta & \psi_{2 p}\left(\boldsymbol{r}_{1}\right) \alpha & \psi_{2 p}\left(\boldsymbol{r}_{1}\right) \beta \\
\psi_{1 s}\left(\boldsymbol{r}_{2}\right) \alpha & \psi_{1 s}\left(\boldsymbol{r}_{2}\right) \beta & \psi_{2 s}\left(\boldsymbol{r}_{2}\right) \alpha & \psi_{2 s}\left(\boldsymbol{r}_{2}\right) \beta & \psi_{2 p}\left(\boldsymbol{r}_{2}\right) \alpha & \psi_{2 p}\left(\boldsymbol{r}_{2}\right) \beta \\
\psi_{1 s}\left(\boldsymbol{r}_{3}\right) \alpha & \psi_{1 s}\left(\boldsymbol{r}_{3}\right) \beta & \psi_{2 s}\left(\boldsymbol{r}_{3}\right) \alpha & \psi_{2 s}\left(\boldsymbol{r}_{3}\right) \beta & \psi_{2 p}\left(\boldsymbol{r}_{3}\right) \alpha & \psi_{2 p}\left(\boldsymbol{r}_{3}\right) \beta \\
\psi_{1 s}\left(\boldsymbol{r}_{4}\right) \alpha & \psi_{1 s}\left(\boldsymbol{r}_{4}\right) \beta & \psi_{2 s}\left(\boldsymbol{r}_{4}\right) \alpha & \psi_{2 s}\left(\boldsymbol{r}_{4}\right) \beta & \psi_{2 p}\left(\boldsymbol{r}_{4}\right) \alpha & \psi_{2 p}\left(\boldsymbol{r}_{4}\right) \beta \\
\psi_{1 s}\left(\boldsymbol{r}_{5}\right) \alpha & \psi_{1 s}\left(\boldsymbol{r}_{5}\right) \beta & \psi_{2 s}\left(\boldsymbol{r}_{5}\right) \alpha & \psi_{2 s}\left(\boldsymbol{r}_{5}\right) \beta & \psi_{2 p}\left(\boldsymbol{r}_{5}\right) \alpha & \psi_{2 p}\left(\boldsymbol{r}_{5}\right) \beta \\
\psi_{1 s}\left(\boldsymbol{r}_{6}\right) \alpha & \psi_{1 s}\left(\boldsymbol{r}_{6}\right) \beta & \psi_{2 s}\left(\boldsymbol{r}_{6}\right) \alpha & \psi_{2 s}\left(\boldsymbol{r}_{6}\right) \beta & \psi_{2 p}\left(\boldsymbol{r}_{6}\right) \alpha & \psi_{2 p}\left(\boldsymbol{r}_{6}\right) \beta
\end{array}\right|
$$


where

$$
\begin{aligned}
& \Psi_{1 s}(\boldsymbol{r})=\left(\frac{\pi a_{0}^{3}}{Z_{e}^{3}}\right)^{-1 / 2} \exp \left(-\frac{Z_{e} r}{a_{0}}\right), \\
& \Psi_{2 s}(\boldsymbol{r})=\frac{1}{\sqrt{4 \pi}}\left(\frac{2 a_{0}}{Z_{e}}\right)^{-3 / 2}\left(2-\frac{Z_{e} r}{a_{0}}\right) \exp \left(-\frac{Z_{e} r}{2 a_{0}}\right), \\
& \Psi_{2 p}(\boldsymbol{r})=\frac{1}{\sqrt{32 \pi}}\left(\frac{Z_{e}}{a_{0}}\right)^{5 / 2} r \exp \left(-\frac{Z_{e} r}{2 a_{0}}\right) \cos (\theta),
\end{aligned}
$$

$\alpha$ and $\beta$ represent spin wave functions for up-spin and down-spin state, respectively. The normalised wave functions $\Psi_{1 s}(\boldsymbol{r}), \Psi_{2 s}(\boldsymbol{r})$, and $\Psi_{2 p}(\boldsymbol{r})$ are orthogonal to each other [20]. The constants $Z_{e}$ in (6a), (6b), and (6c) show the orbital-screening parameter, depending both on the number of bound electrons and on the atomic number $Z_{1}$ of projectile.

The $E_{1 s}$ and $E_{2 s}$ energies, $V_{1 s-1 s}, V_{1 s-2 s}$, and $V_{2 s-2 s}$ direct Coulomb integrals, $A_{1 s-2 s}$ the exchange integral between electrons with parallel spins for $s$-level were calculated by Kaneko [19]. Furthermore, the $E_{2 p}$ energy, $V_{1 s-2 p}, V_{2 s-2 p}$, and $V_{2 p-2 p}$, and $A_{1 s-2 p}$ and $A_{2 s-2 p}$ have been calculated in this study. These are:

$$
\begin{aligned}
& E_{2 p}=\frac{1}{4} E_{1 s}, \\
& V_{1 s-2 p}=\frac{59}{243} e^{2} \frac{Z_{e}}{a_{0}}, \\
& V_{2 s-2 p}=\frac{83}{512} e^{2} \frac{Z_{e}}{a_{0}}, \\
& V_{2 p-2 p}=\frac{501}{2560} e^{2} \frac{Z_{e}}{a_{0}}, \\
& A_{1 s-2 p}=\frac{112}{6561} e^{2} \frac{Z_{e}}{a_{0}}, \\
& A_{2 s-2 p}=\frac{15}{512} e^{2} \frac{Z_{e}}{a_{0}} .
\end{aligned}
$$

Thus, Kaneko's results for $s$-level [19] and results in this study for $p$-level are used to calculate the electronic stopping power and orbital-screening parameters of boron-like and carbon-like swift ions. Using Eqs. (6a-6b), the expectation values $\langle H\rangle_{\mathrm{He}},\langle H\rangle_{\mathrm{Li}}$, and $\langle H\rangle_{\mathrm{Be}}$ of $H$ and orbital-screening parameters $Z_{\mathrm{He}}, Z_{\mathrm{Li}}$, and $Z_{\mathrm{Be}}$ of $Z_{e}$ for helium-like $\left(\xi=2,1 s^{2}\right.$ configuration), lithium-like $\left(\xi=3,1 s^{2} 2 s^{1}\right.$ configuration) and beryllium-like ( $\xi=4,1 s^{2} 2 s^{2}$ configuration) electron systems were calculated by Kaneko [19], respectively. In this study, Eqs. (6a-6c) were used to calculate the expectation values $\langle H\rangle$ of $H$ for carbon-like and boron-like fast ions. 
The expectation value $\langle H\rangle$ of $H$ for carbon-like $\left(\xi=6,1 s^{2} 2 s^{2} 2 p^{2}\right.$ configuration) swift ions is given as

$$
\begin{gathered}
\langle H\rangle_{\mathrm{C}}=2 E_{1 s}+2 E_{2 s}+2 E_{2 p}+V_{1 s-1 s}+4 V_{1 s-2 s}+4 V_{1 s-2 p}+V_{2 s-2 s} \\
+4 V_{2 s-2 p}+V_{2 p-2 p}-2 A_{1 s-2 s}-2 A_{1 s-2 p}-2 A_{2 s-2 p} .
\end{gathered}
$$

If every term is calculated separately and substituted in Eq. (8), respectively, after simple algebra, the total energy for the carbon-like system is represented as

$$
\langle H\rangle_{\mathrm{C}}=\frac{3}{2} Z_{e}^{2}-3 Z_{1} Z_{e}+\frac{55319836}{16796160} Z_{e}
$$

in units of $e^{2} / a_{0}$. This energy takes the minimum value

$$
\langle H\rangle_{\mathrm{C}}=-\frac{3}{2} Z_{\mathrm{C}}^{2}
$$

at $Z_{e}=Z_{\mathrm{C}}$, where

$$
Z_{\mathrm{C}}=\mathrm{Z}_{1}-1.0978 \text {. }
$$

Similarly, we can obtain the total energy expression $\langle H\rangle_{\mathrm{B}}$ for boron-like ( $\xi=5,1 s^{2} 2 s^{2} 2 p$ configuration) electron system as follows:

$$
\begin{aligned}
\langle H\rangle_{\mathrm{B}} & =2 E_{1 s}+2 E_{2 s}+E_{2 p}+V_{1 s-1 s}+4 V_{1 s-2 s}+2 V_{1 s-2 p}+V_{2 s-2 s} \\
& +2 V_{2 s-2 p}-2 A_{1 s-2 s}-A_{1 s-2 p}-A_{2 s-2 p}, \\
\langle H\rangle_{\mathrm{B}} & =\frac{11}{4}\left(Z_{e}^{2}-Z_{1} Z_{e}\right)+\frac{7841956}{3359232} Z_{2}
\end{aligned}
$$

in units of $e^{2} / a_{0}$. The minimum value of (13) can readily be found to be

$$
\langle H\rangle_{\mathrm{B}}=-\frac{11}{8} Z_{\mathrm{B}}^{2}
$$

at $Z_{e}=Z_{\mathbf{B}}$, where

$$
Z_{\mathrm{B}}=\mathrm{Z}_{1}-0.8489 \text {. }
$$

\subsection{Stopping power formula}

The stopping power formulae derived by Kaneko [19] for partially stripped lithium-like and beryllium-like ions were extended to boron-like and carbon-like ions in this study. As explained in the introduction, it is assumed that the charge state of bound electrons of the carbon-like and boron-like projectiles is fixed and not subjected to excitation inside the target material.

As a quantum-mechanical, a general expression for the energy loss formula per unit path length in Born approximation is represented by [21, 22]

$$
S=N \sum_{n}\left(E_{n}-E_{0}\right) \int_{q \min }^{q \max }\left(\frac{\mathrm{d} q}{q^{3}}\right) 8 \pi\left(\frac{e^{2}}{\hbar v}\right) \times\left|F_{00}^{\mathrm{P}}(-\boldsymbol{q})\right|^{2}\left|F_{n 0}^{\mathrm{t}}(\boldsymbol{q})\right|^{2} .
$$


In the above, $E_{n}$ and $E_{0}$, denote the eigen-energies of the target states $n$ and 0 , respectively. $N$ is the number of target atoms per unit volume. The momentum $q$ transferred to the target electrons ranges from $q_{\min }=\left(E_{n}-E_{0}\right) / v$ to $q_{\max }=2 m v$. In Eq. (16) the form factor of projectile, $F_{00}^{\mathrm{p}}(-q)$, and inelastic scattering amplitude of target atom, $F_{n 0}^{\mathrm{t}}(\boldsymbol{q})$, are expressed as

$$
\begin{aligned}
& F_{00}^{\mathrm{p}}(-\boldsymbol{q})=Z_{1}-\left\langle\Psi\left|\sum_{i} \exp \left(\mathrm{i} \boldsymbol{q} \cdot \boldsymbol{r}_{i}\right)\right| \Psi\right\rangle, \\
& F_{n 0}^{\mathrm{t}}(\boldsymbol{q})=\left\langle n\left|\sum_{j} \exp \left(\mathrm{i} \boldsymbol{q} \cdot \boldsymbol{r}_{j}\right)\right| 0\right\rangle .
\end{aligned}
$$

The term $\left\langle\Psi\left|\sum_{i} \exp \left(+\mathrm{i} \boldsymbol{q} \cdot \boldsymbol{r}_{i}\right)\right| \Psi\right\rangle$ on the right hand side of Eq. (17a) [afterwards denoted as $\rho(\boldsymbol{q})$ ] shows the Fourier transform of the spatial electron distribution $\left\langle\Psi\left|\sum_{i} \delta\left(r-r_{i}\right)\right| \Psi\right\rangle$. The charge density of boron-like and carbon-like projectiles are given as

$$
\rho(\boldsymbol{q})=N_{1 s} \rho_{1 s}(\boldsymbol{q})+N_{2 s} \rho_{2 s}(\boldsymbol{q})+N_{2 p} \rho_{2 p}(\boldsymbol{q}),
$$

where $N_{1 s}, N_{2 s}$, and $N_{2 p}$ are the number of the $1 s, 2 s$, and $2 p$ electrons on the projectile, respectively. $N_{1 s}=2$, and $N_{2 s}=2$ for carbon and boron, but $N_{2 p}=1$ for boron and $N_{2 p}=2$ for carbon. The $\rho_{1 s}$ and $\rho_{2 s}$ charge densities for lithium-like and beryllium-like projectiles were found by Kaneko [19]. In this study, $\rho_{2 p}$ charge density has been found to be

$$
\rho_{2 p}(\boldsymbol{q})=\frac{Z_{e}^{8} / a_{0}^{8}-5 q^{2} Z_{e}^{6} / a_{0}^{6}}{\left(q^{8}+4 q^{6} Z_{e}^{2} / a_{0}^{2}+6 q^{4} Z_{e}^{4} / a_{0}^{4}+4 q^{2} Z_{e}^{6} / a_{0}^{6}+Z_{e}^{8} / a_{0}^{8}\right)} .
$$

Substituting $\rho_{1 s}, \rho_{2 s}$, and $\rho_{2 p}$ charge densities in Eq. (18), we can obtain an expression for boron-like and carbon-like ions as follows:

$$
\begin{aligned}
\rho(\boldsymbol{q}) & =2\left[1+\left(q a_{0} / Z_{e}\right)^{2}\right]^{-2}+2 \frac{\left[2\left(q a_{0} / Z_{e}\right)^{2}-1\right]\left[\left(q a_{0} / Z_{e}\right)^{2}-1\right]}{\left[1+\left(q a_{0} / Z_{e}\right)^{2}\right]^{4}} \\
& +N_{2 p} \frac{Z_{e}^{8} / a_{0}^{8}-5 q^{2} Z_{e}^{6} / a_{0}^{6}}{\left[q^{8}+4 q^{6} Z_{e}^{2} / a_{0}^{2}+6 q^{4} Z_{e}^{4} / a_{0}^{4}+4 q^{2} Z_{e}^{6} / a_{0}^{6}+Z_{e}^{8} / a_{0}^{8}\right]} .
\end{aligned}
$$

In order to calculate the electronic stopping power, it is convenient to divide the integration region $\left[q_{\min }, q_{\max }\right]$ into two sections that are $A=\left[q_{\min }, q_{0}\right]$ and $B=\left[q_{0}, q_{\max }\right]$, where $q_{0}$ is a parameter such that the dipole approximation can be used for $F_{n 0}^{\mathrm{t}}(\boldsymbol{q})$. Physically, this means the separation of close and distant collisions. Hereby, we can obtain $\exp \left(-\mathrm{i} \boldsymbol{q} \cdot \boldsymbol{r}_{j}\right)=1-\mathrm{i} \boldsymbol{q} \cdot \boldsymbol{r}_{j}$ for $\boldsymbol{q} \cdot \boldsymbol{r}_{j} \ll 1$ and the contribution of the distant collision, $S_{A}$, is then represented by

$$
S_{A}=N \sum_{n}\left(E_{n}-E_{0}\right) 8 \pi\left(\frac{e^{2}}{\hbar v}\right)^{2}\left|d_{n 0}\right|^{2} \times \int_{q_{\min }}^{q_{0}}\left(\frac{\mathrm{d} q}{q}\right)\left|F_{00}^{\mathrm{p}}(-q)\right|^{2}
$$

Here $d_{n 0}$ is the dipole-transition matrix element. On the other hand, the contribution of the close collision, $S_{B}$, is given by 


$$
S_{B}=N\left(\frac{\hbar^{2}}{2 m}\right) Z_{2} 8 \pi\left(\frac{e^{2}}{\hbar v}\right)^{2} \times \int_{q_{0}}^{q_{\max }}\left(\frac{\mathrm{d} q}{q}\right)\left|F_{00}^{\mathrm{p}}(-\boldsymbol{q})\right|^{2} .
$$

Here we can interchange the order of the summation over $n$ and the integration over $q$ because both $q_{\max }$ and $q_{0}$ are independent of the eigenstate $|n\rangle$. Hence, we can use the sum rule $[20,23]$.

$$
\sum_{n}\left(E_{n}-E_{0}\right)\left|F_{n 0}^{\mathrm{t}}(q)\right|^{2}=\left(\frac{\hbar^{2} q^{2}}{2 m}\right) Z_{2} .
$$

The definite integrals on the right hand side in $S_{A}$ and $S_{B}$ can be estimated if we used the following result:

$$
\int \frac{\mathrm{d} q}{q}\left|F_{00}^{\mathrm{p}}(-\boldsymbol{q})\right|^{2}=\int \frac{\mathrm{d} q}{q}\left[Z_{1}-\rho(\boldsymbol{q})\right]^{2} .
$$

Substituting Eq. (20), if this integral is calculated, Eq. (24) is found to be

$$
\begin{aligned}
& \int \frac{\mathrm{d} q}{q}\left[Z_{1}-\rho(\boldsymbol{q})\right]^{2}=A \ln (q)+B_{0} \ln \left[\left(q a_{0} / Z_{e}\right)^{2}+1\right] \\
& \quad+\sum_{n=1}^{7} B_{n} /\left[\left(q a_{0} / Z_{e}\right)^{2}+1\right]^{n}+C_{0} \ln \left[\left(q a_{0} / Z_{e}\right)^{2}+4\right] \\
& \quad+\sum_{n=1}^{3} C_{n} /\left[\left(q a_{0} / Z_{e}\right)^{2}+4\right]^{n}
\end{aligned}
$$

Here integral constants are:

$$
\begin{aligned}
& A=\left(Z_{1}-N_{2 p}-4\right)^{2} \\
& B_{0}=2 Z_{1}+Z_{1} N_{2 p}-(98 / 81) N_{2 p}-(1 / 2) N_{2 p}^{2}+350 / 81, \\
& B_{1}=-Z_{1} N_{2 p}-2 Z_{1}+(278 / 27) N_{2 p}+(1 / 2) N_{2 p}^{2}+758 / 27 \\
& B_{2}=Z_{1}-(1 / 2) Z_{1} N_{2 p}-(13 / 3) N_{2 p}+(1 / 4) N_{2 p}^{2}-151 / 9 \\
& B_{3}=-4 Z_{1}-2 Z_{1} N_{2 p}+(70 / 9) N_{2 p}+(1 / 6) N_{2 p}^{2}+134 / 9 \\
& B_{4}=(1 / 2) N_{2 p}+(1 / 8) N_{2 p}^{2}-3 / 2, \quad B_{5}=(22 / 5) N_{2 p}+(1 / 10) N_{2 p}^{2}+10, \\
& B_{6}=-12 N_{2 p}-2 N_{2 p}^{2}-16, \quad B_{7}=(72 / 7) N_{2 p}+(18 / 7) N_{2 p}^{2}+72 / 7 \\
& C_{0}=2 Z_{1}-(226 / 81) N_{2 p}-998 / 81, \quad C_{1}=(56 / 27) N_{2 p}-8 Z_{1}+152 / 9 \\
& C_{2}=16, \quad C_{3}=128 / 3 . \quad
\end{aligned}
$$


For the distant collision, $S_{A}$, the upper limit $\left(q=q_{0}\right)$ and lower limit $\left(q=q_{\min }\right)$ are replaced in Eq. (25) and in $q_{\min }^{2}\left(a_{0} / Z_{e}\right)^{2} \ll 1$ approximation and then we obtain

$$
\begin{aligned}
S_{A} & =\left(\frac{4 \pi e^{4}}{m v^{2}}\right) N Z_{2}\left\{2 A \ln \left(q_{0}\right)+B_{0} \ln \left[1+\left(q_{0} a_{0} / Z_{e}\right)^{2}\right]\right. \\
& +\sum_{n=1}^{7} B_{n} /\left[1+\left(q_{0} a_{0} / Z_{e}\right)^{2}\right]^{n}+C_{0} \ln \left[4+\left(q_{0} a_{0} / Z_{e}\right)^{2}\right] \\
& +\sum_{n=1}^{3} C_{n} /\left[4+\left(q_{0} a_{0} / Z_{e}\right)^{2}\right]^{n}-2 A \ln \left(q_{\min }\right)-B_{0}\left(q_{\min } a_{0} / Z_{e}\right)^{2} \\
& -\sum_{n=1}^{7} B_{n} /\left[1+n\left(q_{\min } a_{0} / Z_{e}\right)^{2}\right]-C_{0}\left[\ln (4)+\left(q_{\min } a_{0} / 2 Z_{e}\right)^{2}\right] \\
& \left.-\sum_{n=1}^{3} C_{n}\left[\left(1 / 4^{n}\right)-\left(n / 4^{n+1}\right)\left(q_{\min } a_{0} / Z_{e}\right)^{2}\right]\right\} .
\end{aligned}
$$

For the close collision, $S_{B}$, the upper limit $\left(q=q_{\max }\right)$ and lower limit $\left(q=q_{0}\right)$ are written in Eq. (25) and in $q_{\max }^{2}\left(a_{0} / Z_{e}\right)^{2} \gg 4$ approximation and then we get

$$
\begin{aligned}
S_{B}= & \left(\frac{4 \pi e^{4}}{m v^{2}}\right) N Z_{2}\left\{2 A \ln \left(q_{\max }\right)+B_{0}\left[\ln \left(q_{\max } a_{0} / Z_{e}\right)^{2}+\left(1 /\left(q_{\max } a_{0} / Z_{e}\right)^{2}\right]\right.\right. \\
& +B_{1} /\left(q_{\max } a_{0} / Z_{e}\right)^{2}+C_{0}\left[\ln \left(q_{\max } a_{0} / Z_{e}\right)^{2}+4 /\left(q_{\max } a_{0} / Z_{e}\right)^{2}\right] \\
& -\sum_{n=1}^{7} B_{n} /\left[1+\left(q_{0} a_{0} / Z_{e}\right)^{2}\right]^{n}-C_{0} \ln \left[4+\left(q_{0} a_{0} / Z_{e}\right)^{2}\right] \\
& \left.-\sum_{n=1}^{3} C_{n} /\left[4+\left(q_{0} a_{0} / Z_{e}\right)^{2}\right]^{n}\right\} .
\end{aligned}
$$

As the contributions of the distant and close collisions are separately evaluated and constants are replaced, the sum of $S_{A}$ and $S_{B}$ gives the total electronic stopping power for boron-like and carbon-like ions.

$$
S=\left(4 \pi e^{2} / m v^{2}\right) N Z_{2} L\left(Z_{1}, Z_{2}, v\right)
$$

Here $L\left(Z_{1}, Z_{2}, v\right)$ is the number of stopping and as shown 


$$
\begin{aligned}
& L\left(Z_{1}, Z_{2}, v\right)=\left(Z_{1}-N_{2 p}-4\right)^{2} \ln \left(2 m v^{2} / I\right) \\
& \quad+\left[2 Z_{1} N_{2 p}-(196 / 81) N_{2 p}-N_{2 p}^{2}+4 Z_{1}+700 / 81\right] \ln \left(2 v / Z_{e} v_{0}\right) \\
& \quad+4\left[Z_{1}-(113 / 81) N_{2 p}-499 / 81\right] \ln \left(v / Z_{e} v_{0}\right)+7 Z_{1}+(7 / 2) Z_{1} N_{2 p} \\
& \quad-(32971 / 1890) N_{2 p}-13177 / 378-(1439 / 840) N_{2 p}^{2},
\end{aligned}
$$

where $I$ denotes the mean excitation energy of the target material. In Eq. (28b), for boron-like particles, $Z_{e}=Z_{1}-0.8488$ and $N_{2 p}=1$ and for carbon-like particles, $Z_{e}=Z_{1}-1.0978$ and $N_{2 p}=2$. Here, the separation parameter $q_{0}$ vanishes.

It is seen that the stopping power of the target material is characterised by three quantities $\left(I, Z_{2}\right.$, and $\left.N\right)$. It is interesting that the first term of $(28 \mathrm{~b})$ is interpreted as Bethe's original form for a net charge $\left(Z_{1}-N_{2 p}-4\right)$. This term comes from the logarithmic term in Eq. (25). The spatial size of the projectile is completely neglected there. The other terms are correction terms.

\subsection{The concept of effective charge in electronic stopping power}

In this section, the affection of effective charge of the projectile will be discussed. The concept of effective charge is very useful to understand and compile the stopping power data. This thought is based on the proportionality of the Bethe formula to the square of the charge of projectile. The effective charge is defined as the square root of the ratio of the stopping power $S$ to the proton stopping power $S_{\mathrm{p}}$ at the same velocity:

$$
Z_{\mathrm{eff}}=\left(S / S_{\mathrm{p}}\right)^{1 / 2}
$$

The view of the effective charge is to summarise various effects on the electronic excitation such as the spatial size and charge-changing effects into only one parameter $Z_{\text {eff }}$. As used the definition of $Z_{\text {eff }}$, the quantity $L\left(Z_{1}, Z_{2}, v\right)$ of Eq. (28b) is given by $\left(Z_{\text {eff }}\right)^{2} \ln \left(2 m v^{2} / I\right)$.

The effective charge has generally two aspects that are charge-exchange and spatial size effects. The charge-exchange is represented by stripping bound electrons more and more to become a bare nucleus with increasing velocity. On the other hand the spatial size effect becomes larger the contribution of close collisions, where the target electrons are scattered by an incident particle with effective charge greater than its net charge. The reason is the incomplete screening of the nuclear charge by bound electrons. In the frozen charge state ions, the distribution of electrons is assumed to be fixed during passing even at high velocities. In this case, the effective charge of frozen charge state ions contains only the size effect.

Let us show an exact representation for $Z_{\text {eff }}$ of ions in a frozen charge state. From Eq. (28b) and the definition of $Z_{\text {eff }}$, we can easily show for boron-like and carbon-like ions by 


$$
\begin{aligned}
Z_{\mathrm{eff}}^{2} & =\left(Z_{1}-N_{2 p}-4\right)^{2}+\left[\ln \left(2 m v^{2} / I\right)\right]^{-1} \\
& \times\left\{\left[2 Z_{1} N_{2 p}-(196 / 81) N_{2 p}-N_{2 p}^{2}+4 Z_{1}+700 / 81\right] \ln \left(2 v / Z_{e} v_{0}\right)\right. \\
& +4\left[Z_{1}-(113 / 81) N_{2 p}-499 / 81\right] \ln \left(v / Z_{e} v_{0}\right)+7 Z_{1}+(7 / 2) Z_{1} N_{2 p} \\
& \left.-(32971 / 1890) N_{2 p}-13177 / 378-(1439 / 840) N_{2 p}^{2}\right\} .
\end{aligned}
$$

Here $Z_{1}$ is the atomic number of an incident ion, $N_{2 p}$ is the number of electrons in $2 p$ orbital and $v$ is the velocity of the incident ion.

In the high velocity limit, i.e., when $v \gg Z_{e} v_{0}$ and $v \gg(I / 2 m)^{1 / 2}, Z_{\text {eff }}$ in Eq. (30) approaches the asymptotic value

$$
Z_{\text {eff }}^{2}=\frac{1}{2}\left[\left(Z_{1}-N_{2 p}-4\right)^{2}+Z_{1}^{2}\right]
$$

which is independent of velocity.

Equation (31) means that in the high velocity limit the square of the effective charge is the arithmetic average of the square of the nuclear charge of a projectile and square of the net charge [24]. This relation shows us that there are equal contributions from two extreme cases, i.e. complete neglect of bound electrons and complete screening of the nucleus.

When the effective charge $Z_{\text {eff }}(q)$ of an ion in the charge state $q\left(=Z_{1}-N_{1 s}-\right.$ $N_{2 s}-N_{2 p}$ ) is derived from Eq. (30), the magnitude of screening can be estimated by a bound electron. Here we can define the screening charge $Z_{\mathrm{sc}}(q, q-1)$ by

$$
Z_{\mathrm{sc}}(q, q-1)=Z_{\mathrm{eff}}(q)-Z_{\mathrm{eff}}(q-1)
$$

This screen charge will be found to show the shell effect to the stopping power [19].

\section{Results and discussion}

Here, we discuss first the validity of the choice of wave function. It is seen that it is quite reasonable [25] to describe the ground-state boron-like $\left(1 s^{2} 2 s^{2} 2 p\right)$ and carbon-like $\left(1 s^{2} 2 s^{2} 2 p^{2}\right)$ projectiles by $s$ - and $p$-types wave functions. The total energy of the system calculated in this way should be compared with any available detailed results but we could not find a good example, so a comparison was only made for neutral boron and carbon atoms. According to (9) and (10), and (13) and (14), the calculating total energy of the neutral boron and carbon atoms are $E=-23.693$ and $E=-36.046 \mathrm{eV}$, respectively. However, Clementi and Roetti [25] calculated $E_{\mathrm{CR}}=-24.529 \mathrm{eV}$ for boron atom and $E_{\mathrm{CR}}=-37.688 \mathrm{eV}$ for carbon atoms, so that the difference $\Delta E=E-E_{\mathrm{CR}}=0.836$ and $\Delta E=E-E_{\mathrm{CR}}=1.642$ for boron and carbon atom, respectively. This is caused by a rather rough description of the one-electron wave function adopted. However, the ratio $\Delta E / E_{\mathrm{CR}}$ amounts for boron and carbon atoms to only 3.40 and $4.356 \%$, respectively. Furthermore, orbital screening parameters for boron and carbon atoms are 4.1511 and 
4.9021 in our calculation and 4.2236 and 5.0125 in Clementi and Roetti's one [25]. Then, according to the obtained results in this study, we think the trial wave function in the present variational method is not bad. But the values of ratio $\Delta E / E_{\mathrm{CR}}$ increase with increasing the atomic number of projectiles. Therefore, it can be said that this method does not work in heavier atoms.

Figures 1, 2, and 3 show the calculated electronic energy loss of carbon for $\mathrm{O}^{q+}(q=2-3), \mathrm{N}^{q+}(q=1-2)$, and $\mathrm{C}^{q+}(q=0-1)$ ions, respectively, with velocity from $v=3 v_{0}$ to $v=90 v_{0}$ under the frozen-charge state condition. Here, the mean excitation energy $I$ of carbon is taken to be $I=77.3 \mathrm{eV}$ [1].

We used equations (28a) and (28b) in calculation of the electronic energy loss for $\mathrm{O}^{3+}$ (B-like) and $\mathrm{O}^{2+}$ (C-like), $\mathrm{N}^{2+}$ (B-like) and $\mathrm{N}^{1+}$ (C-like) and $\mathrm{C}^{1+}$ (B-like) and $\mathrm{C}$ ions, respectively. In general, the average radius of the bound electron becomes smaller with increasing $Z_{1}$ because it is approximately inversely proportional to $Z_{1}$. As a result, the net-charge approximation is valid for heavier (or larger $Z_{1}$ ) ions because of the screening of the projectile's nuclear charge by the bound electrons. Equation (28) clearly shows that the first term, $\ln \left(2 m v^{2} / I\right)$, plays a dominant role for large $Z_{1}$. In other words, the ion can be considered as a point charge. This picture leads to the other conclusion that the effective stopping-power charge $Z_{\text {eff }}$ reduces to the net charge. As a result, the effective charge depends on both $Z_{1}$ and ion velocity. The electronic energy loss of $\mathrm{O}^{2+}$ ion is smaller than $\mathrm{O}^{3+}$ one at a higher velocity.

Figures 1, 2, and 3 show that the stopping power decreases at a higher energy region as velocity increases. Also, the difference between the stopping power values for boron-like and carbon-like ions increases with increasing the atomic number of the incident ion.

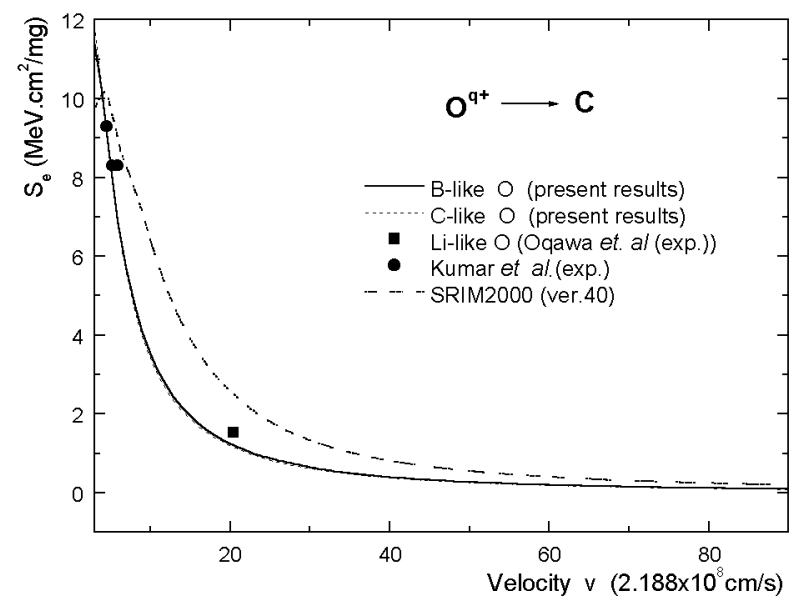

Fig. 1. Electronic stopping power of carbon for $\mathrm{O}^{q+}(q=2-3)$ ions as a function of velocity. The theoretical results of Eq. (28) are drawn for $q=2$, B-like, $q=3$, C-like. The experimental data are taken form Ogawa et al. [27] and Kumar [28]. For comparison, we also show SRIM2000 calculated results [26]. 
Figure 1 shows the calculated stopping power of carbon for $\mathrm{O}^{q+}(q=2-3)$ ions with a velocity from $v=3 v_{0}$ to $v=90 v_{0}$, together with SRIM2000 results [26] and experimental data for lithium-like oxygen of Ogawa et al. [27] at $v=20.6 v_{0}$ under the frozen-charge-state condition and for oxygen ions of Kumar [28]. Ogawa et al. have found that the fixed charge stopping powers of carbon for $169.4 \mathrm{MeV}$

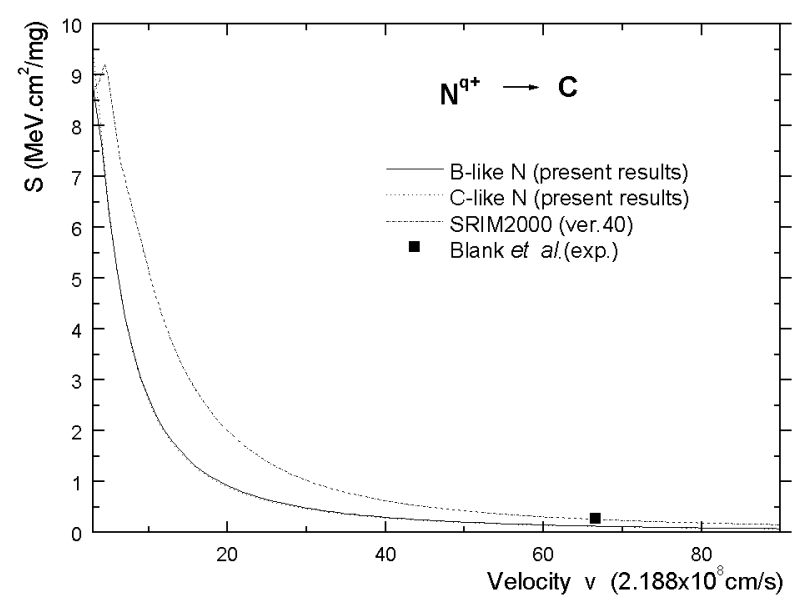

Fig. 2. Electronic stopping power of carbon for $\mathrm{N}^{q+}(q=1-2)$ ions as a function of velocity. The present theoretical results of Eq. (28) are drawn for $q=1$, B-like; $q=2$, C-like. We also show SRIM2000 calculated results [26]. The experimental data are taken from Blank et al. [29].

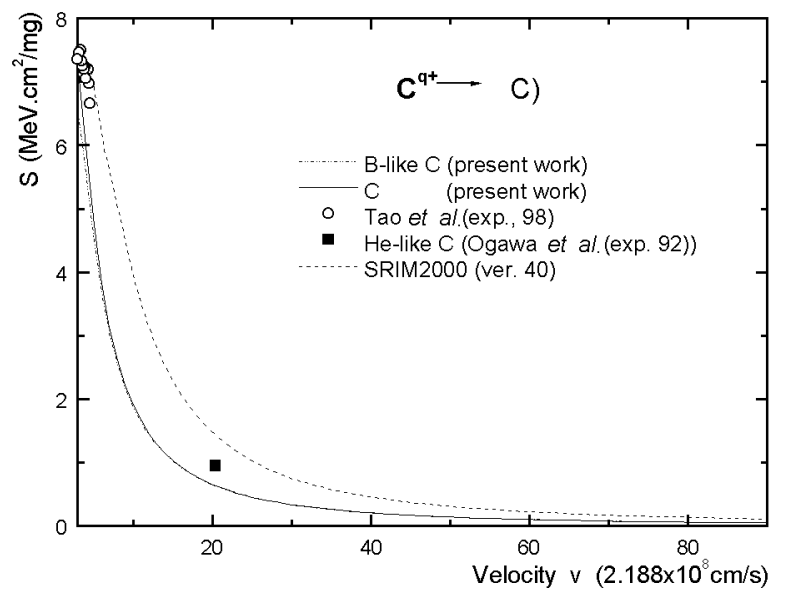

Fig. 3. Electronic stopping power of carbon for $\mathrm{C}^{q+}(q=0-1)$ ions as a function of velocity. The theoretical results of Eq. (28) are drawn for $q=1$ B-like; $q=0$, C-like. We also show SRIM2000 calculated results [26]. The experimental results are taken from Tao et al. [30] and for He-like C ions from Ogawa et al. [31]. 
oxygen ions are $2.617 \pm 0.023 \mathrm{keV} /\left(\mu \mathrm{g} / \mathrm{cm}^{2}\right), 2.171 \pm 0.018 \mathrm{keV} /\left(\mu \mathrm{g} / \mathrm{cm}^{2}\right), 1.797 \pm$ $0.024 \mathrm{keV} /\left(\mu \mathrm{g} / \mathrm{cm}^{2}\right)$ and $1.517 \pm 0.027 \mathrm{keV} /\left(\mu \mathrm{g} / \mathrm{cm}^{2}\right)$ for $\mathrm{O}^{8+}, \mathrm{O}^{7+}, \mathrm{O}^{6+}$, and $\mathrm{O}^{5+}$, respectively. According to this, the stopping power for oxygen at the same energy decreases as $q$ decreases. Therefore, the present calculation results are consistent with these experimental results.

Figure 2 shows the calculated stopping power of carbon for $\mathrm{N}^{q+}(q=1-2)$ ions with a velocity from $v=3 v_{0}$ to $v=90 v_{0}$, together with SRIM2000 results [26] and experimental data [29]. The calculated stopping power results for beryllium-like nitrogen and carbon-like $\mathrm{N}$ is lower than SRIM2000 results, because in the present work, the electronic stopping power for $\mathrm{N}$ has been calculated for B-like ions with five bound electrons and C-like ions with six bound electrons.

Figure 3 shows the calculated stopping power of carbon for $\mathrm{C}^{q+}(q=0-1)$ ions with a velocity from $v=3 v_{0}$ to $v=90 v_{0}$, together with SRIM2000 results [26] and experimental data $[30,31]$. Experimental energy loss data for a helium-like carbon ion are taken from Ogawa et al. [31]. Agreement between the calculated electronic energy loss results from Eq. (28) and the experimental data is good, but lower than the SRIM2000 calculated results.

Figures 4,5 , and 6 show the effective charge $Z_{\text {eff }}$ calculated for $\mathrm{O}^{q+}$ $(q=2-3), \mathrm{N}^{q+}(q=1-2)$, and $\mathrm{C}^{q+}(q=0-1)$ ions, respectively, in collisions with carbon targets at a velocity from $v=3 v_{0}$ to $v=90 v_{0}$. From the Figures, it can be seen that the effective charge of a particularly stripped ion increases with a velocity in any charge state. At very high velocities, the effective charge is at least saturated, to be constant given by Eq. (30) or (32). The Figures show the importance of the effect of bound electrons attached to a projectile even at high velocities.

In this study, as a result, the ground state energies have been calculated by using hydrogen-like wave functions for boron and carbon ions $(\xi \leq 6)$. After that,

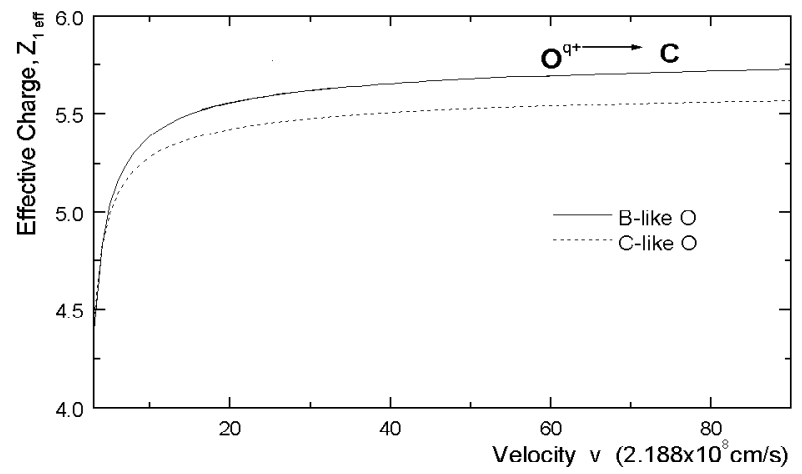

Fig. 4. Effective charge of $\mathrm{O}^{q+}(q=2-3)$ ions passing through carbon foils as a function of velocity. 


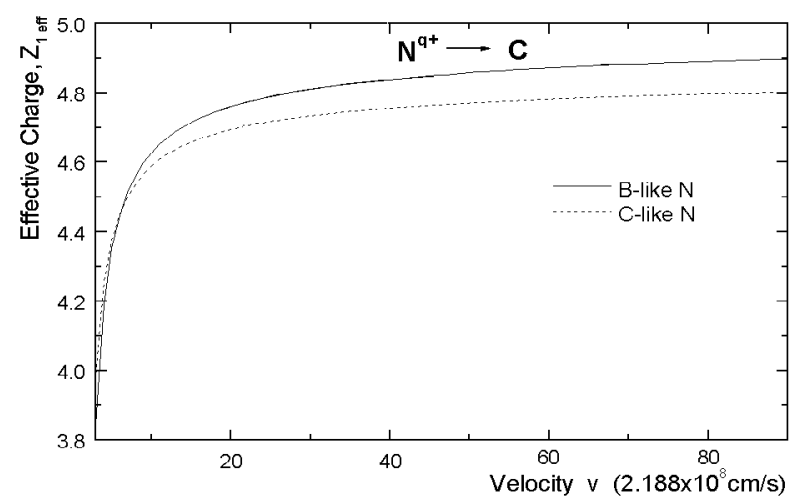

Fig. 5. Effective charge of $\mathrm{N}^{q+}(q=1-2)$ ions passing through carbon foils as a function of velocity.

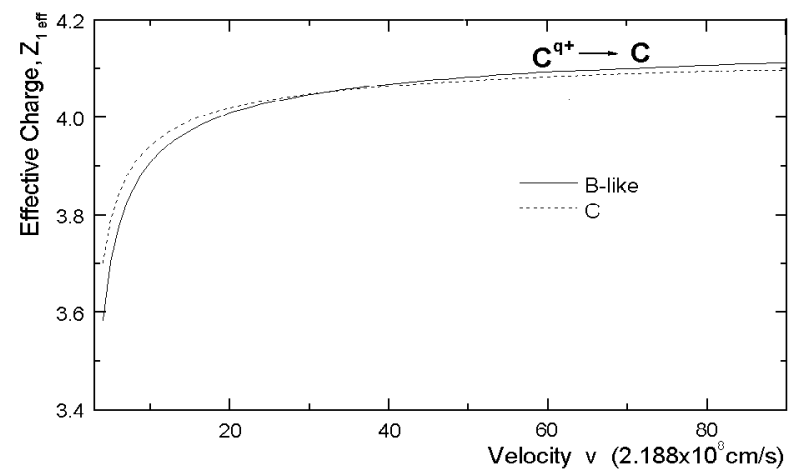

Fig. 6. Effective charge of $\mathrm{C}^{q+}(q=0-1)$ ions passing through carbon foils as a function of velocity.

$Z_{e}$ variation parameters were found for this. The calculated ground state total energies are consistent with the values of Clementi-Roetti with $3-4 \%$.

In conclusion, the analytical expression for the electronic stopping power for boron-like and carbon-like projectiles in a frozen-charge state was derived on the basis of the first-order perturbation theory and the Hartree-Fock-Slater method. Also, the correction term $\Delta L\left(Z_{1}, Z_{2}, v\right)$ at a high velocity can be easily obtained from these expressions. The effective stopping power charge of frozen-charge-state projectiles was calculated.

\section{References}

[1] H.H. Anderson, J.F. Ziegler, The Stopping and Ranges of Ions in Matter, Pergamon, New York 1977.

[2] J.F. Janni, At. Data Nucl. Data Tables 27, 147 (1982). 
[3] H.A. Bethe, Ann. Phys. (Leipzig) 5, 325 (1930).

[4] F. Bloch, Ann. Phys. (Leipzig) 16, 285 (1933).

[5] R.H. Rithche, Phys. Rev. 114, 644 (1959).

[6] J. Neufeld, R.H. Ritchie, Phys. Rev. 98, 1632 (1955).

[7] J. Lindhard, A. Winther, K. Dan. Vidensk. Selsk. Mat. Fys. Medd. 34, No. 4 (1964).

[8] P. Sigmund, Phys. Rev. A 26, 2497 (1982).

[9] P.M. Echenique, R.M. Nieminen, J.C. Ashley, R.H. Ritchie, Phys. Rev. A 33, 897 (1986).

[10] I. Gertner, M. Meron, B. Rosner, Phys. Rev. A 18, 2022 (1978); 21, 1191 (1980).

[11] T. Kaneko, Phys. Rev. A 30, 1714 (1984); 33, 1602 (1986).

[12] T. Kaneko, Phys. Rev. A 40, 2188 (1989); Phys. Status Solidi B 156, 49 (1989).

[13] W.H. Barkas, N.J. Dyer, H.H. Heckman, Phys. Rev. Lett. 11, 26 (1963).

[14] M.C. Walske, Phys. Rev. 88, 1283 (1952); 101, 940 (1956).

[15] T.L. Ferrell, H.H. Ritchie, Phys. Rev. B 16, 115 (1977).

[16] W. Brant, M. Kitagawa, Phys. Rev. B 25, 5631 (1982).

[17] T. Kaneko, Phys. Rev. A 41, 4889 (1990); Nucl. Instrum. Methods B 48, 83 (1990).

[18] T. Kaneko, Phys. Rev. A 43, 4780 (1991).

[19] T. Kaneko, Phys. Rev. A 49, 4780 (1994).

[20] See, for example, L.D. Landau, E.M. Lifshitz, Quantum Mechanics, Pergamon, London 1958.

[21] Y.-K. Kim, K.-T. Cheng, Phys. Rev. A 22, 61 (1980).

[22] G.H. Gillespie, M. Inokuti, Phys. Rev. A 22, 2430 (1980).

[23] H.A. Bethe, E.E. Salpeter, Quantum Mechanics of One- and Two Electron Atoms, Plenium, New York 1977.

[24] O.H. Crawford, Phys. Rev. A 39, 4432 (1989).

[25] E. Clementi, C. Roetti, At. Data Nucl. Data Tables 14, 177 (1974).

[26] J.F. Ziegler, J.P. Biersack, SRIM2000-, Stopping and Range of Ions in Matter, IBM Research, Yorktown Heights (NY) 105981, USA.

[27] H. Ogawa, I. Kitayama, I. Sugai, Y. Haruyama, N. Saita, K. Yoshida, M. Tosaki, H. Ikegami, Nucl. Instrum. Methods B 82, 80 (1993).

[28] V. Hari Kumar, A.P. Pathak, S.K. Sharma, S. Kumar, N. Nath, D. Kabiraj, D.K. Avasti, Nucl. Instrum. Methods B 108, 223 (1996).

[29] B. Blank, J.J. Gaimard, H. Geissel, G. Münzberg, K.H. Schmidt, H. Seltzer, K. Summerer, Nucl. Instrum. Methods B 51, 85 (1990).

[30] Z. Tao, Lu. Xiting, Z. Yongjun, Xia Zonhuang, S. Dingyu, W. Xuemei, Z. Qiang, Nucl. Instrum. Methods B 135, 169 (1998).

[31] H. Ogawa, I. Kitayama, I. Sugai, Y. Haruyama, M. Tosaki, A. Aoki, K. Yoshida, H. Ikegami, Phys. Lett. A 167, 487 (1993). 